\title{
A comparison of the risk of cesarean section in gestational diabetes mellitus patients supplemented antenatally with vitamin D containing supplements versus placebo: A systematic review and meta-analysis of double-blinded randomized controlled trials
}

\author{
(D) Sumanta Saha1, (D) Sujata Saha² \\ ${ }^{1}$ National Institute for Research in Tuberculosis, India \\ 2Mankar College, India
}

\section{Abstract}

\begin{abstract}
The aim of this study was to study the role of vitamin D containing supplements in the risk of cesarean section (CS), a common complication in gestational diabetes mellitus (GDM) patients. An additional objective was to assess the risk of developing pre-eclampsia, preterm delivery, macrosomia, and polyhydramnios in these participants. Various electronic databases were searched for double-blinded parallel-arm randomized controlled trials that reported the incidence of CS in adult, non-insulin treated GDM patients who received vitamin D and placebo in different treatment arms, respectively. Next, each eligible trial's risk of bias was assessed, and the effects of the above interventions on the respective outcomes were compared meta-analytically across the trials. This review included five Iranian trials sourcing data from nearly 380 participants. The risk of bias in the trials was primarily low. In contrast to the placebo group, the risk of CS [risk ratio (RR): $0.61, p=0.002,95 \%$ confidence interval (CI): 0.44,0.83; $\mathrm{I}^{2}=0 \%$, p-value of Cochrane's Q: 0.373 ) and macrosomia (RR: $0.31, \mathrm{p}=0.006,95 \%$ CI: $0.13,0.72 ; \mathrm{I}^{2}=0 \%$, p-value of Cochrane's Q: 0.935] was less in the vitamin D supplemented group. The remaining outcomes did not differ between the intervention groups. The antenatal use of vitamin D containing supplements in non-insulin treated GDM patients might reduce the risk of CS and macrosomia. (J Turk Ger Gynecol Assoc 2020; 21: 201-12)
\end{abstract}

Keywords: Diabetes, gestational, vitamin D, cesarean section, fetal macrosomia, pre-eclampsia, premature birth, polyhydramnios

Received: 05 October, 2019 Accepted: 20 April, 2020

\section{Introduction}

Gestational diabetes mellitus (GDM) is a glucose intolerance to any degree occurring at the start of pregnancy or first recognized during gestation (1). It is diagnosed between 2428 weeks of gestation using screening tests with a 50 gram and 1-hour glucose challenge test (1). It is classified as either A1GDM or A2GDM, depending on whether it is managed with dietary therapy or medication, respectively (1). The chief medication used to treat GDM if diet and exercise therapy fails is insulin (1). Glyburide and metformin, two oral hypoglycemic agents with the potential to cross the placenta, are also used to treat GDM frequently. However, such use of these medications is not approved by the U.S. Food and Drug Administration due to inadequate safety information $(1,2)$. Unlike type 1 and type 2 diabetes, newer drugs such as sodium-glucose linked transporter 2 inhibitors, remain poorly studied in GDM patients (3-5). 
GDM can cause both neonatal complications including macrosomia, neonatal hypoglycemia, shoulder dystocia, and hyperbilirubinemia and maternal complications $(1,6)$. One of the chief maternal complications of GDM is cesarean section (CS), in which the fetus is delivered surgically by incising the abdomen and uterus of the parturient (1,7-9). The prevalence of CS is high in GDM patients (32-44\%), and it is more common than in parturients with no glucose intolerance $(7,10-15)$. The indication for CS is determined by the obstetric need of the GDM mother and includes indications such as preeclampsia, macrosomia, excessive fetal growth (fetal weight more than $4500 \mathrm{gm}$ ), and past obstetric history, for example previous history of childbirth by CS (7,8,16-18). CS increases the risk of wound hematoma, anesthetic complications, major puerperal infection, and severe hemorrhage which may result in hysterectomy (19). Moreover, women undergoing planned vaginal delivery are less likely to have severe morbidity or mortality compared to those delivered by CS on an emergency basis (19).

To minimize these surgical risks, it is important to identify new pragmatic treatment options that can decrease the incidence of CS in GDM patients. In this regard, the plausible clinical role of antenatal vitamin D supplementation in GDM patients is a novel area to explore, as suggested by recent vitamin D-related research. Existing studies suggest a possible association between vitamin D deficiency and GDM (20-24). Moreover, GDM prevalence tends to decrease on prenatal supplementation of vitamin D (25,26). Besides, maintaining the recommended optimum vitamin D status during pregnancy might be protective against CS, although the mechanism remains unclear (27-29). When vitamin D is complemented in GDM patients, it facilitates better glycemic control when measured by a decrease in fasting plasma glucose and/or insulin and improvement in homeostasis model of assessment-insulin resistance (20-24,30,31). All these vitamin D related findings in pregnancy and GDM formed the rationale for undertaking this study; to explore the risk of CS in (antenatal) vitamin D supplemented GDM patients.

\section{The intervention}

Vitamin D is a fat-soluble hormone (32). It is available from diet and supplements in two physiologically inactive forms - D2 (ergocalciferol) and D3 (cholecalciferol) (33,34). Vitamin D3 is additionally synthesized in the skin on exposure to the sun (33). The active form of vitamin D, calcitriol 1,25-(dihydroxyvitamin) 2D, is produced on hydroxylation of vitamin D2 and D3 successively in the liver and kidneys $(33,35)$. This active form plays a role in the physiology of pregnancy via the vitamin D receptors in uteroplacental tissue $(33,35)$.

Recently, different clinical trials have tested the health effects of antenatal vitamin D supplementation in GDM patients.
However, the route of vitamin D administration [parenteral (36) versus oral (37-40)], dosing, and the accompanying supplements (when used) varied among such trials. Some trials in pregnant women have used vitamin D as a sole supplement, (36-38) while others used it with co-supplements such as magnesium, zinc, or calcium $(39,40)$. A trial that tested the role of intramuscular administration of vitamin D in GDM patients, used it as a single injection of 300.000 IU (36). In clinical trials that prescribed oral vitamin D, GDM patients were advised to take it at a dose of 50.000 IU, 2-3 weeks apart for 3-8 weeks $(38,40)$. Other such trials asked GDM patients to take 200-500 IU of oral vitamin D twice daily for 6-16 weeks $(37,41)$.

\section{What this review adds?}

In GDM patients, the contemporary evidence of the effect of antenatal vitamin D supplementation on CS, and other obstetric outcomes are based on the evidence of clinical trials, like those reviewed in this paper. However, to the best of our knowledge, there has been no previous attempt to synthesize the overall rigor of such evidence by systematic review and meta-analysis. Therefore, this paper reviews this poorly evidenced area of GDM literature and synthesizes new evidence based on the existing highest quality of epidemiological studies (i.e., doubleblinded randomized clinical trials). In addition, as this study involved GDM mothers who were not on insulin treatment, the latter's therapeutic effects are unlikely to bias this findings of this study.

\section{Aim}

This study aimed to compare the risk of CS between noninsulin treated GDM patients supplemented antenatally with vitamin D containing supplements and placebo. The auxiliary objective was to compare the risk of macrosomia, polyhydramnios, pre-eclampsia, and pre-term delivery among these treatment groups.

\section{Material and Methods}

Inclusion criteria: 1. Study design: Parallel-arm (any number of arms) double-blinded randomized controlled clinical trials of any duration were eligible. 2. Participant: The eligible participants were adult (18 years or older) females diagnosed with GDM by American Diabetes Association criteria $(42,43)$, between 24-28 weeks of their concurrent pregnancy who received the intervention of interest before initiation of insulin therapy. 3. Intervention compared: The above-described trials should compare the following interventions - vitamin D (in D2 or D3 form or both; as a sole supplement or adjunct to any other supplements) with placebo. Vitamin D supplementation was accepted irrespective of its dose and route of administration; 
oral or intramuscular. 4. Outcome: The trials must report the frequency of $\mathrm{CS}$ observed in each of the studied treatment groups, post-intervention.

Exclusion criteria: 1 . Study design: Differing from that described in the inclusion criteria, which included observational study designs, single-arm interventional studies, and cross-over trials. 2. Participants: With diabetes of any other type except GDM or those diagnosed previously with GDM were excluded from this review.

The secondary outcomes of interest were the risk of macrosomia, polyhydramnios, pre-eclampsia, and pre-term delivery. However, these did not contribute to the inclusion criteria. This review follows the PRISMA (44) reporting guideline and does not have a pre-published protocol.

The search for eligible trials was conducted in electronic databases (PubMed, Embase, and Scopus) with no restriction to date or language. The following search strategy was used in PubMed: "vitamin D" or calciferol OR "vitamin D2" or ergocalciferol or "vitamin D3" or cholecalciferol or cholecalciferol (MeSH) or "ergocalciferols" (MeSH) AND "diabetes, gestational" (MeSH) and "gestational diabetes" or GDM. The search was restricted to clinical trials by using the filters "Clinical Trial" and "Randomized Controlled Trial." Identical search terms were used for searching the other databases. The last date of database search was 07 February, 2020.

The papers identified by the electronic database search were skimmed for trials matching this review's eligibility criteria. Publications were read in full text when they seemed to match these criteria or in circumstances where a decision of their inclusion or exclusion was not possible by reading the titles and abstracts only. Besides the above, an auxiliary search was conducted in the references of the papers that were included in this review.

Then the following data were extracted from the included trials: author information (first author's last name and year of publication), study design (randomization, blinding, if placebo-controlled, single or multicentric, funding, ethical clearance, trial ID), participants (diagnosis, gestational age of GDM diagnosis, number randomized, mean age, participant consent, trial nation), interventions (intervention/s received by each of the trial arms), and outcomes. Using the appropriate tool from the Cochrane Collaboration, the risk of selection bias in the trials (based on random sequence generation and concealment of participant allocation), performance bias, detection bias, attrition bias, reporting bias and miscellaneous bias were assessed and categorized as high risk, low risk, and unclear risk (45).

The first author conducted the database search and retrieved the eligible trials and their data. The co-author subsequently rechecked it. The risk of bias in the respective trials was assessed by each author independently, and then the findings were cross referenced and matched. The authors resolved disputes in their opinion at all stages of this review by discussion. The intervention effects on the outcomes were compared across the trials by the random-effect model meta-analysis (DerSimonian and Laird) method, and the summary effect was determined in risk ratios (RR). Despite the relative homogeneity of the participant characteristics and study design, a randomeffect model was used since the vitamin D supplement adjuncts used between the trails were not identical. To determine the effects of vitamin D as a chief supplement, in trials that used it in multiple treatment arms, we chose one that included a fewer number of vitamin D adjuncts. For meta-analyses, when an outcome occurred in one of the intervention arms of a trial only, 0.5 was added to each cell of the $2 \times 2$ table. Heterogeneity was assessed using the p-value of Cochranes Q (statistical significance determined at $\mathrm{p}<0.1)$ in conjunction with $\mathrm{I}^{2}$ statistics $(0-40 \%, 30-60 \%, 50-90 \%$, and $75-100 \%$ represented less, moderate, substantial, and considerable heterogeneity, respectively) (45). Funnel plots were used to visually assess publication bias.

Finally, sensitivity analyses were performed, in which the metaanalysis for the respective outcomes was iterated using a fixedeffect model (inverse-variance method) and also by excluding a study each time (using both fixed-effect and random effect model). At $\mathrm{p}<0.05$ and 95\% confidence interval, results were considered statistically significant. The Stata statistical software (StataCorp, College Station, Texas, USA; version 16) was used to perform statistical analyses.

\section{Results}

The initial electronic search returned 836 citations. After excluding the duplicates, the titles and abstracts of 757 papers were read. For 16 studies, full-text reading ensued. Finally, five trials meeting the eligibility criteria of this review were included for the risk of bias assessment and quantitative analysis (Figure 1) (46-50). These trials were published between 2015-19, were primarily single centered (47-51) except one (46), and based on about $380 \mathrm{GDM}$ patients from Iran. The average age of these participants was approximately between 28-32 years (46-50). Two of these trials $(48,50)$ tested vitamin D as a sole supplement in one of their treatment arms (48). In the intervention arms of the remaining trials, vitamin D was co-supplemented with another supplement including probiotics, magnesium, calcium, and zinc $(46,47,49)$. All trials had a placebo arm (46-50). Each trial reported both the primary and secondary outcomes (46$50)$. 


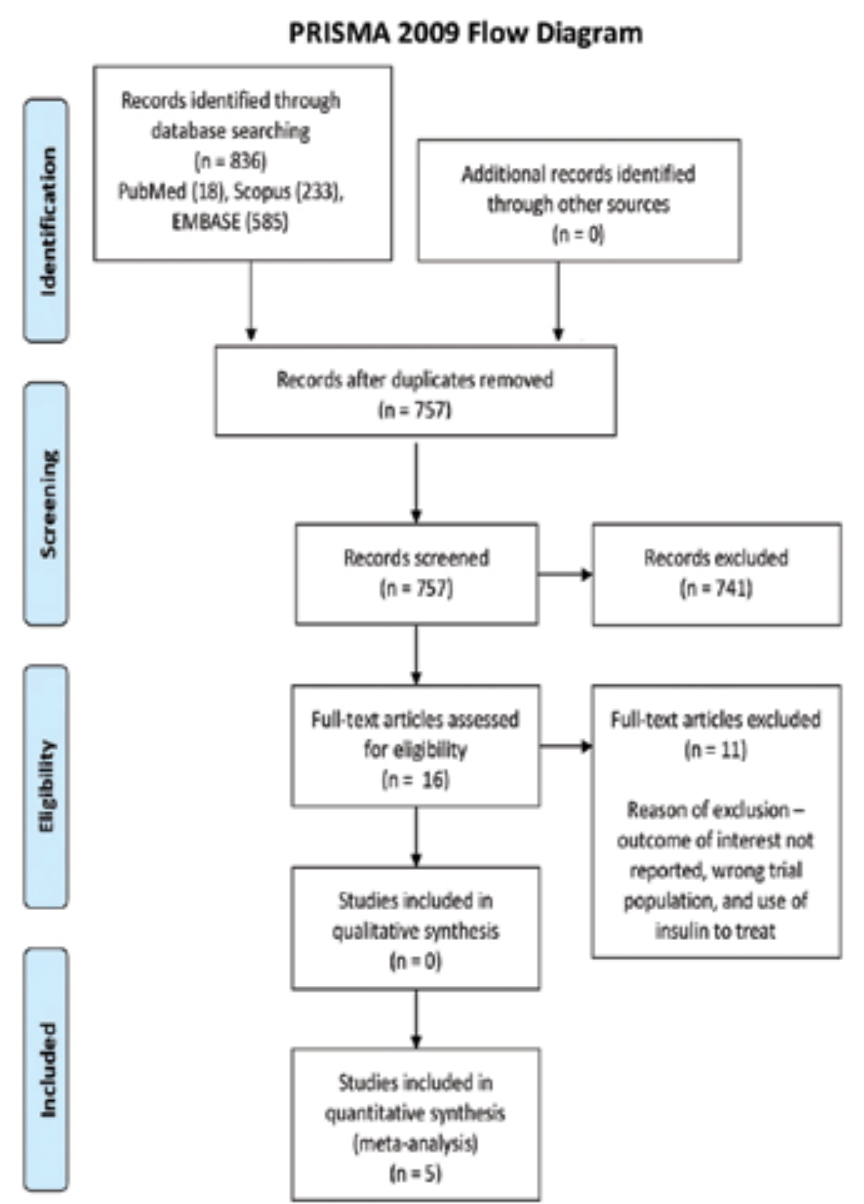

Figure 1. PRISMA 2009 Flow Diagram [From: Moher D, Liberati A, Tetzlaff J, Altman DG, The PRISMA Group (2009). Preferred Reporting Items for Systematic Reviews and MetaAnalyses: The PRISMA Statement. PLoS Med 6(7): e1000097. doi:10.1371/journal.pmed1000097]

Regarding the appraisal of the studies, overall the trials are at a low risk of bias except for unclear risk of allocation concealment in four trials $(46,47,49,50)$ and performance bias in one trial (47). Table 1 presents the salient features and the risk of bias assessment of the reviewed trials (46-50).

Upon meta-analysis, GDM patients receiving vitamin D containing supplements had a lower risk of experiencing CS (RR: $0.61, p=0.002,95 \%$ confidence interval (CI): 0.44,0.83; $\mathrm{I}^{2}=0 \%$, p-value of Cochrane's Q: 0.373 ) and macrosomia (RR: $0.31, p=0.006,95 \%$ CI: $0.13,0.72 ; I^{2}=0 \%$, p-value of Cochrane's $\mathrm{Q}: 0.935)$ than the placebo recipients. The risk of the remaining outcomes did not vary between the compared interventions. Overall, for all outcomes, statistical heterogeneity was classified as less, that is between $0-40 \%$ (45). The forest plots (Figure 2-6) depict the outcome data along with their effect sizes.

On visual inspection, the funnel plots (not shown) were not suggestive of any publication bias. Sensitivity analysis results were almost identical to the preliminary analyses (Table 2).

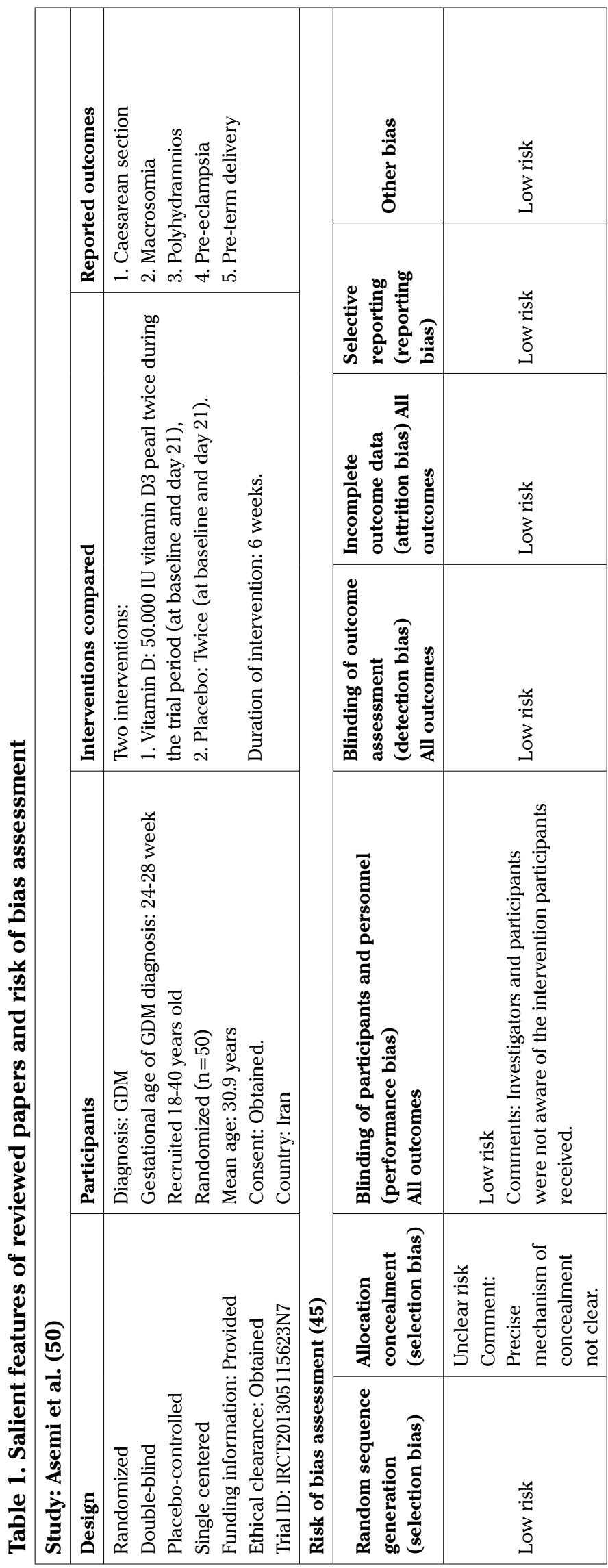




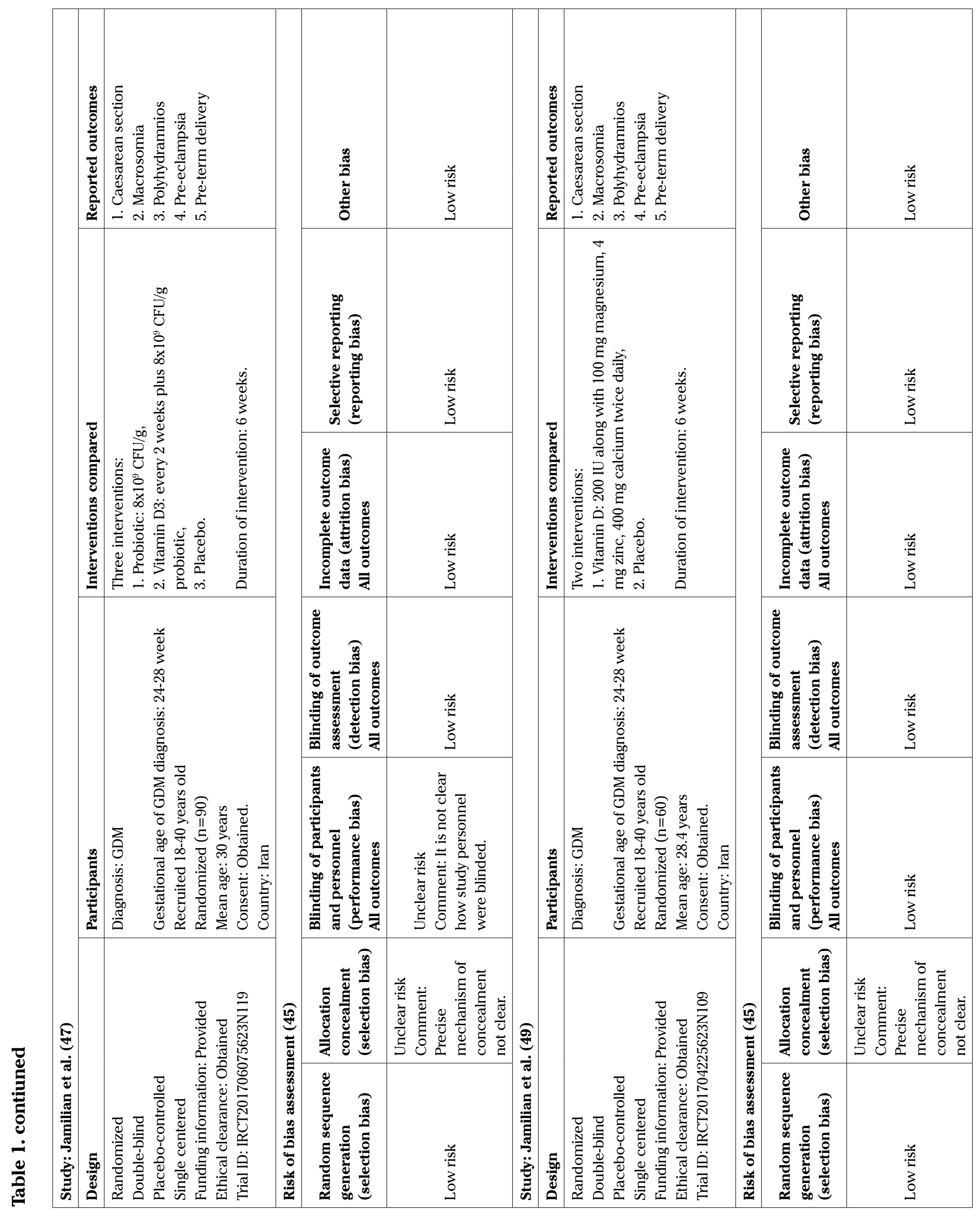




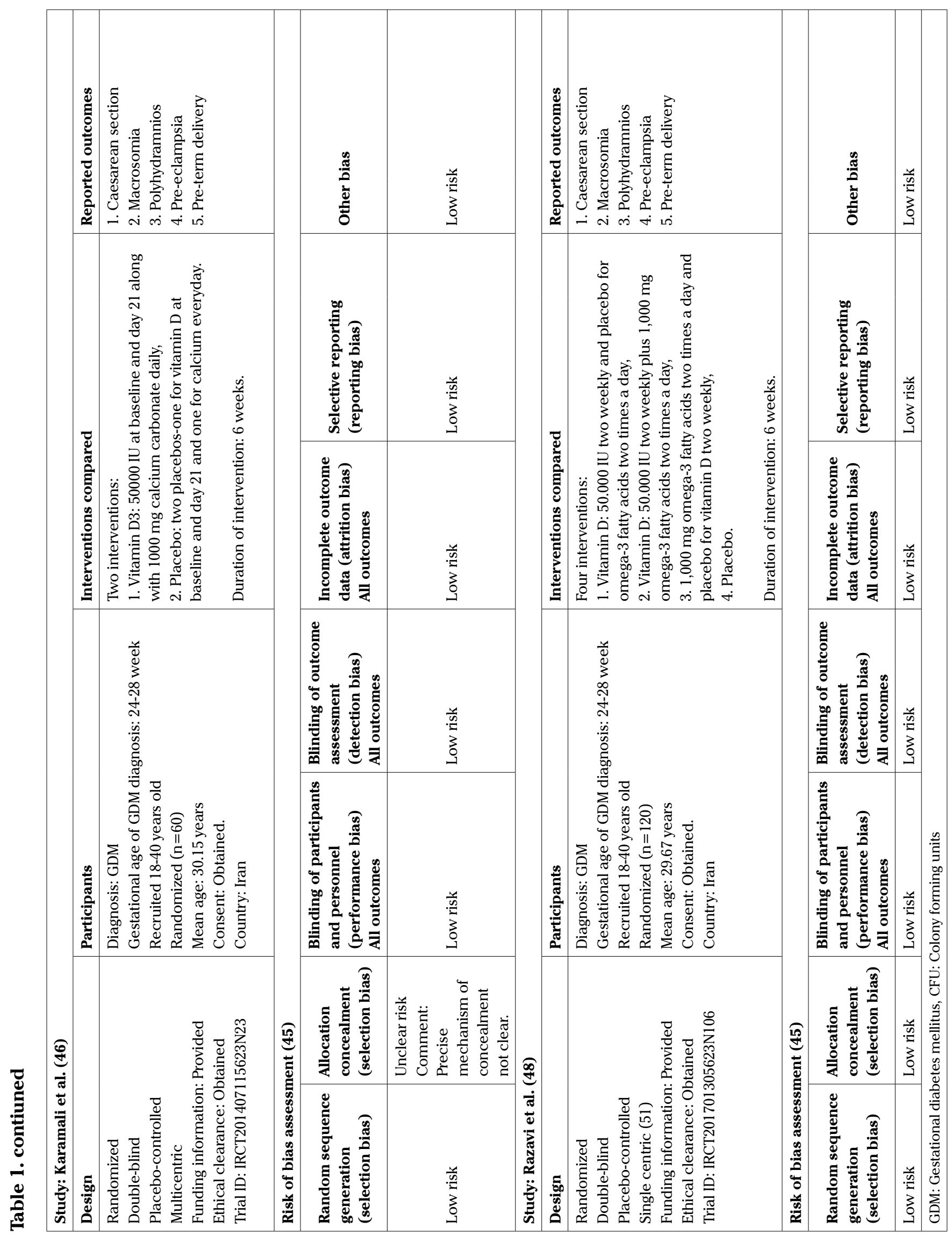


Table 2. Sensitivity analysis (by dropping a trial in each meta-analytic iteration)

\begin{tabular}{|c|c|c|c|c|c|c|c|}
\hline \multirow[b]{2}{*}{ Outcome } & \multicolumn{2}{|l|}{ Dropped study } & \multicolumn{2}{|l|}{ RR $(95 \%$ CI) } & \multirow[b]{2}{*}{$\mathbf{p}$} & \multicolumn{2}{|c|}{ Heterogeneity } \\
\hline & Author & Year & RE model & FE model & & $\begin{array}{l}\mathbf{I}^{2} \text { statistics } \\
(\%)\end{array}$ & $\begin{array}{l}\text { p-value of } \\
\text { Cochrane's Q }\end{array}$ \\
\hline \multirow{5}{*}{$\begin{array}{l}\text { Caesarean } \\
\text { section }\end{array}$} & Asemi et al. (50) & 2015 & $0.53(0.36,0.78)$ & $0.53(0.36,0.78)$ & $0.001 *$ & $0 \%$ & 0.470 \\
\hline & Jamilian et al. (47) & 2019 & $0.61(0.41,0.90)$ & $0.62(0.44,0.87)$ & $0.012 *$ & $19 \%$ & 0.295 \\
\hline & Jamilian et al. (49) & 2019 & $0.62(0.44,0.88)$ & $0.63(0.45,0.87)$ & $0.008^{*}$ & $12.6 \%$ & 0.329 \\
\hline & Karamali et al. (46) & 2016 & $0.69(0.48,0.97)$ & $0.69(0.48,0.97)$ & $0.033^{*}$ & $0 \%$ & 0.708 \\
\hline & Razavi et al. (48) & 2017 & $0.57(0.40,0.80)$ & $0.57(0.40,0.80)$ & $0.001 *$ & $0 \%$ & 0.395 \\
\hline \multirow{5}{*}{ Pre-term delivery } & Asemi et al. (50) & 2015 & $0.65(0.15,2.73)$ & $0.65(0.15,2.73)$ & 0.552 & $0 \%$ & 0.698 \\
\hline & Jamilian et al. (47) & 2019 & $0.66(0.16,2.79)$ & $0.66(0.16,2.79)$ & 0.572 & $0 \%$ & 0.711 \\
\hline & Jamilian et al. (49) & 2019 & $0.65(0.15,2.75)$ & $0.65(0.15,2.75)$ & 0.559 & $0 \%$ & 0.703 \\
\hline & Karamali et al. (46) & 2016 & $0.33(0.07,1.61)$ & $0.33(0.07,1.61)$ & 0.170 & $0 \%$ & 1.000 \\
\hline & Razavi et al. (48) & 2017 & $0.65(0.15,2.75)$ & $0.65(0.15,2.75)$ & 0.559 & $0 \%$ & 0.703 \\
\hline \multirow{5}{*}{ Pre-eclampsia } & Asemi et al. (50) & 2015 & $0.60(0.25,1.45)$ & $0.60(0.25,1.45)$ & 0.258 & $0 \%$ & 0.816 \\
\hline & Jamilian et al. (47) & 2019 & $0.70(0.25,1.92)$ & $0.70(0.25,1.92)$ & 0.482 & $0 \%$ & 0.893 \\
\hline & Jamilian et al. (49) & 2019 & $0.55(0.21,1.47)$ & $0.55(0.21,1.47)$ & 0.233 & $0 \%$ & 0.799 \\
\hline & Karamali et al. (46) & 2016 & $0.60(0.25,1.46)$ & $0.60(0.25,1.46)$ & 0.261 & $0 \%$ & 0.820 \\
\hline & Razavi et al. (48) & 2017 & $0.45(0.16,1.25)$ & $0.45(0.16,1.25)$ & 0.127 & $0 \%$ & 0.957 \\
\hline \multirow{5}{*}{ Polyhydramnios } & Asemi et al. (50) & 2015 & $0.48(0.18,1.26)$ & $0.48(0.18,1.26)$ & 0.136 & $0 \%$ & 0.740 \\
\hline & Jamilian et al. (47) & 2019 & $0.39(0.13,1.19)$ & $0.39(0.13,1.19)$ & 0.099 & $0 \%$ & 0.557 \\
\hline & Jamilian et al. (49) & 2019 & $0.40(0.15,1.09)$ & $0.40(0.15,1.09)$ & 0.072 & $0 \%$ & 0.557 \\
\hline & Karamali et al. (46) & 2016 & $0.49(0.18,1.37)$ & $0.49(0.18,1.37)$ & 0.175 & $0 \%$ & 0.677 \\
\hline & Razavi et al. (48) & 2017 & $0.32(0.11,0.90)$ & $0.32(0.11,0.90)$ & $0.032 *$ & $0 \%$ & 0.795 \\
\hline \multirow{5}{*}{ Macrosomia } & Asemi et al. (50) & 2015 & $0.30(0.12,0.75)$ & $0.30(0.12,0.75)$ & $0.010^{*}$ & $0 \%$ & 0.847 \\
\hline & Jamilian et al. (47) & 2019 & $0.28(0.10,0.78)$ & $0.28(0.10,0.78)$ & $0.014^{*}$ & $0 \%$ & 0.865 \\
\hline & Jamilian et al. (49) & 2019 & $0.33(0.13,0.85)$ & $0.33(0.13,0.85)$ & $0.021^{*}$ & $0 \%$ & 0.889 \\
\hline & Karamali et al. (46) & 2016 & $0.34(0.14,0.82)$ & $0.34(0.14,0.82)$ & $0.017^{*}$ & $0 \%$ & 0.959 \\
\hline & Razavi et al. (48) & 2017 & $0.27(0.10,0.75)$ & $0.27(0.10,0.75)$ & $0.012 *$ & $0 \%$ & 0.882 \\
\hline
\end{tabular}

\section{Discussion}

To summarize, five recent double-blinded randomized controlled Iranian trials (comprising about 380 GDM patients) compared the obstetric risk of CS, macrosomia, polyhydramnios, pre-eclampsia, and pre-term delivery between the prenatal recipients of vitamin $\mathrm{D}$ and placebo. The risk of bias in the trials was predominantly low with occasional unclear risk of bias components (46-50). The meta-analyses suggested that in GDM patients, antenatal vitamin D containing supplement recipients had a reduced risk of CS and macrosomia than those who took a placebo.

The evidence quality of CS and macrosomia was graded using the GRADE approach [GRADE Working Group (2004)] (52). Due to the unclear risk of bias present in some of the trials, the evidence was downgraded by one level to moderate-quality evidence.

The scope of contrasting the findings of this review with the existing literature is limited, due to its conceptual novelty. In this regard, there is a recent review by Cochrane collaboration comparing obstetric outcomes between the vitamin D (as a sole or complementary supplement) and placebo receiving pregnant females (27). It found no major difference in the risk of CS between these intervention groups (27). However, unlike this review, the Cochrane collaboration review (27) was not specific to the GDM subpopulation.

The implications of this review are discussed here. First, healthcare professionals caring for GDM patients might find this review of worth to expand their existing knowledge in this context. Next, research in this milieu may help to 


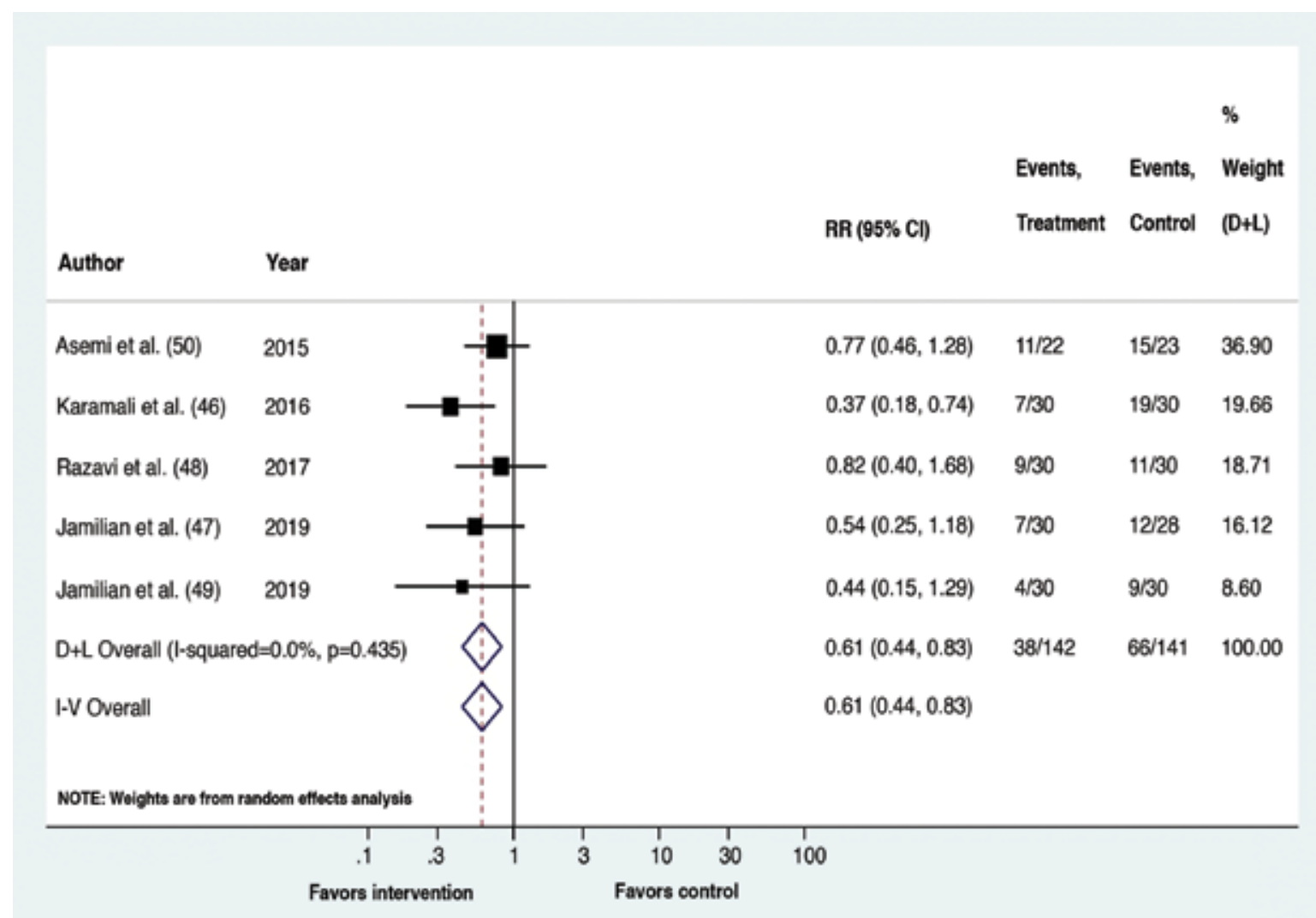

Figure 2. Forest plot: Comparison between vitamin D supplemented group and placebo for the outcome cesarean section

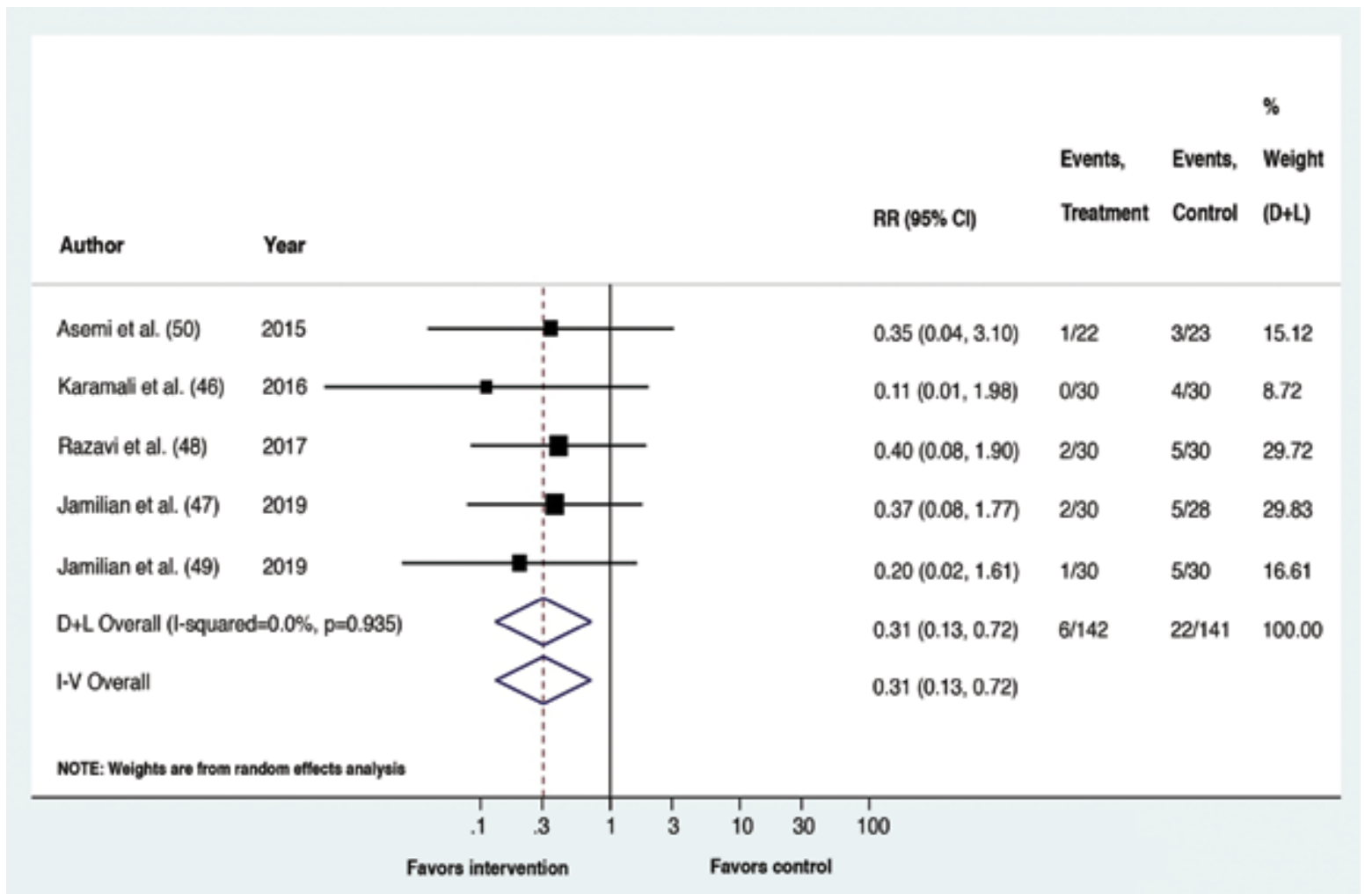

Figure 3. Forest plot: Comparison between vitamin D supplemented group and placebo for the outcome macrosomia 


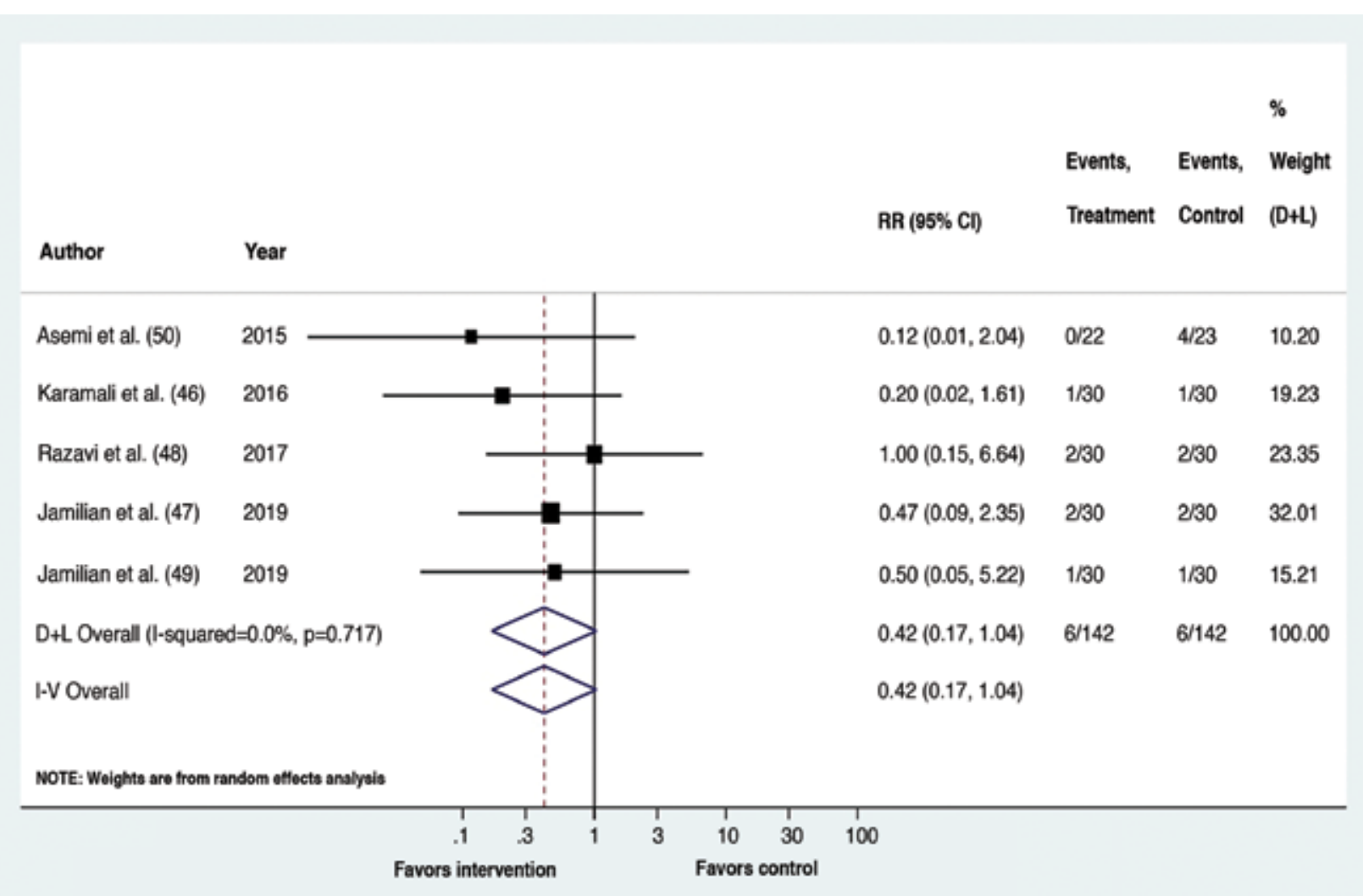

Figure 4. Forest plot: Comparison between vitamin D supplemented group and placebo for the outcome polyhydramnios

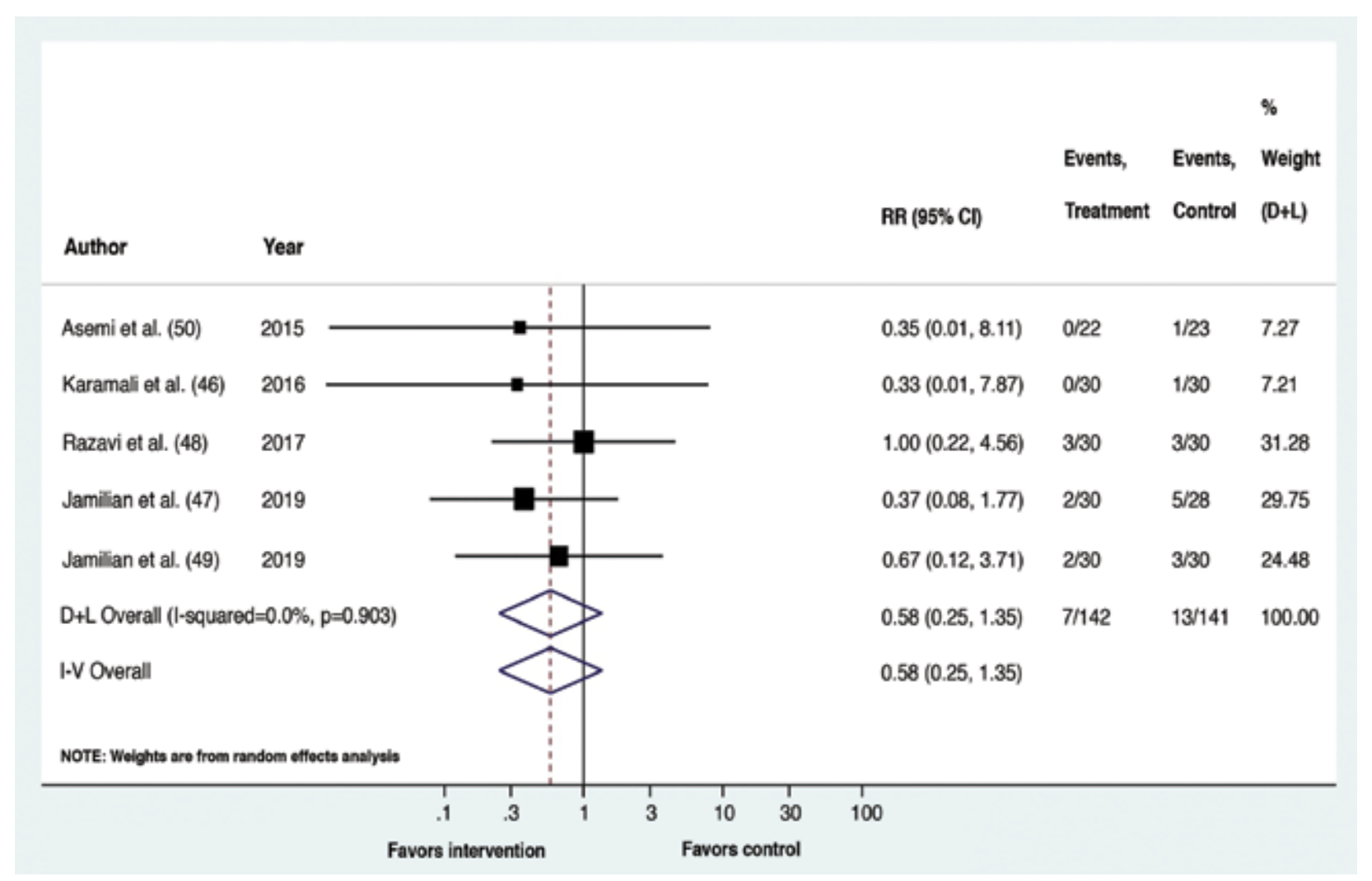

Figure 5. Forest plot: Comparison between vitamin D supplemented group and placebo for the outcome pre-eclampsia 


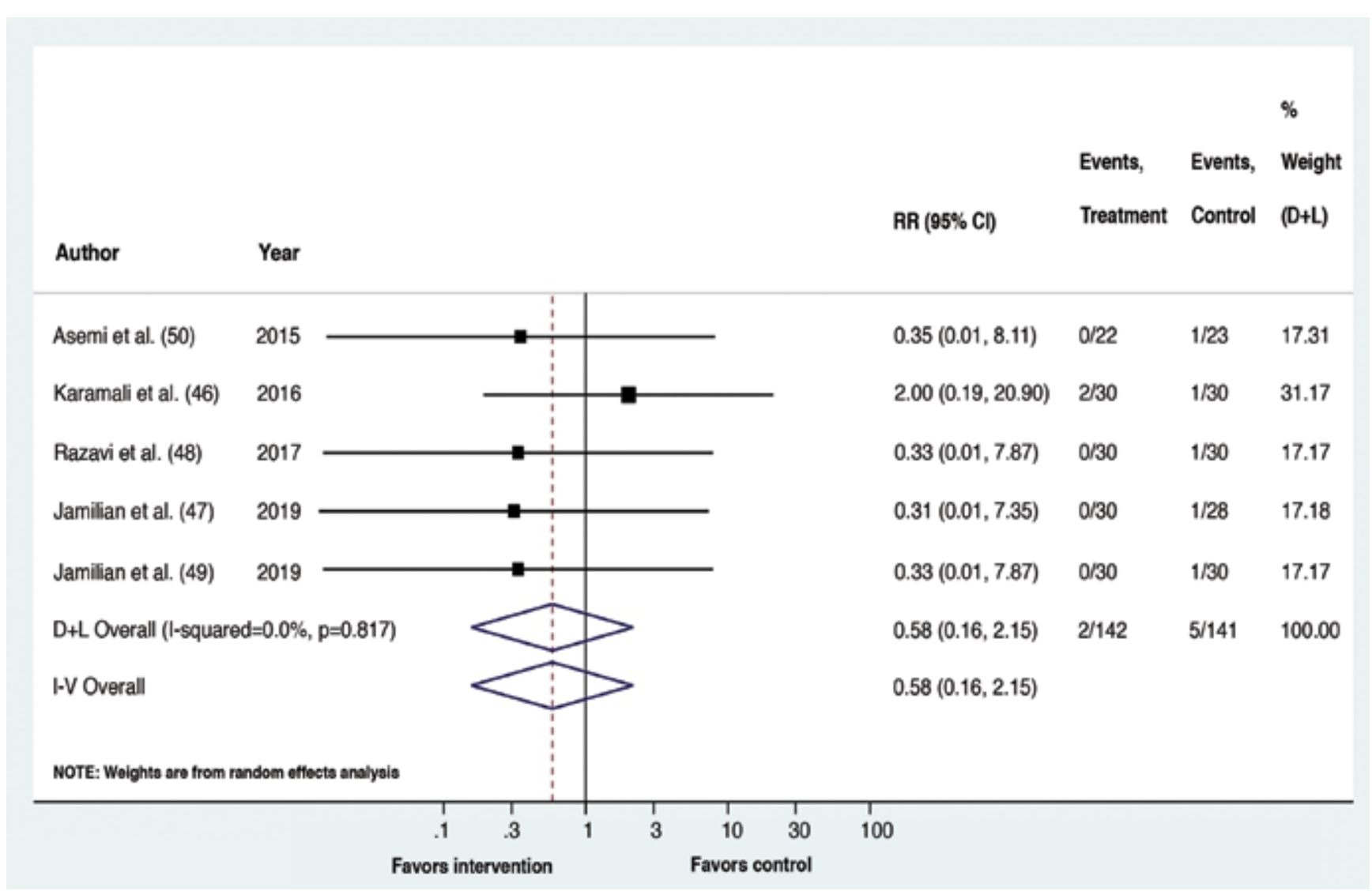

Figure 6. Forest plot: Comparison between vitamin D supplemented group and placebo for the outcome pre-term delivery

inform public health policy about endorsing prenatal vitamin D supplementation in GDM patients. The lower risks of macrosomia and CS due to vitamin D supplementation may encourage future researchers to investigate if there is a causal relationship between these. Moreover, future researchers from nations other than Iran may also consider researching this context to test if these paper's findings are externally valid or not.

The following are the strengths of this review. First, this is perhaps the first systematic review that attempted to synthesize evidence in this study's context. Second, the findings of this review are likely to be rigorous as it utilized evidence from double blinded randomized controlled trials, the highest level of epidemiological evidence. Third, this review is expected to be more comprehensive as its database search method was not restricted to any date or language. Lastly, the meta-analysis findings regarding CS and macrosomia are likely to be robust due to their similarity with the sensitivity analysis.

Despite these strengths, there are certain limitations of this paper. At the review level, the number of trials investigating the context was relatively few, which might have compromised the external validity of this meta-analysis. At the outcome level, by including intervention arms of trials that tested vitamin $D$ along with other nutritional adjuncts, it is difficult to conclude if the observed effects were influenced by the latter. At the study level, the weaknesses were the unclear risk of bias $(46,47,49,50)$, single centric study design (47-50), and relatively small sample size (46-50). Additionally, as all trials were Iranbased (46-50), the findings are unlikely to be generalizable to the global population.

\section{Conclusion}

The contemporary evidence in non-insulin treated GDM patients from Iran suggests that antenatal vitamin $\mathrm{D}$ containing supplements decreases the risk of CS and macrosomia, compared to placebo. However, to increase the external validity of these findings, methodologically rigorous trials from different parts of the globe might be useful in the future. Furthermore, future trials may use vitamin $\mathrm{D}$ as the sole supplement to specifically identify its effects on obstetric outcomes in GDM patients.

Peer-review: Externally peer-reviewed. 
Conflict of Interest: No conflict of interest was declared by the authors.

Financial Disclosure: The authors declared that this study received no financial support.

\section{References}

1. Quintanilla Rodriguez BS, Mahdy H. Gestational Diabetes. StatPearls. 2019.

2. Committee on Practice Bulletins-Obstetrics. ACOG Practice Bulletin No. 190: Gestational Diabetes Mellitus. Obstet Gynecol 2018; 131: 49-64.

3. Mosley JF 2nd, Smith L, Everton E, Fellner C. Sodium-Glucose Linked Transporter 2 (SGLT2) Inhibitors in the Management Of Type-2 Diabetes: A Drug Class Overview. P T 2015; 40: 451-62.

4. Saha S. An Appraisal of a Systematic Review and Meta-Analysis of Randomized Clinical Trials on the Efficacy and Safety of SodiumGlucose Cotransporter-2 Inhibitors as an Adjunct to Insulin Therapy in Type 1 Diabetes Patients. Int J Diabetes Metab 2019: $1-1$.

5. Saha S, Saha S. A Systematic Review and Meta-Analysis of Randomised Controlled Trials, Contrasting the Safety Profile between Sodium-Glucose Cotransporter-2 Inhibitors and Placebo in Type 1 Diabetes Mellitus Patients. Int J Diabetes Metab. 2020: 1-12

6. Saha S. Compliance and barriers to self-monitoring of blood glucose in patients with gestational diabetes mellitus: A systematic review. Int J Health Sci (Qassim). 2019; 13: 44-52.

7. Coustan DR. Gestational Diabetes Mellitus. Clin Chem 2013; 59: 1310-21.

8. Alfadhli E. Gestational diabetes mellitus. Saudi Med J 2015; 36: 399406.

9. Sung S, Mahdy H. Cesarean Section. StatPearls. 2019.

10. Boriboonhirunsarn D, Waiyanikorn R. Emergency cesarean section rate between women with gestational diabetes and normal pregnant women. Taiwan J Obstet Gynecol 2016; 55: 64-7.

11. Prakash GT, Das AK, Habeebullah S, Bhat V, Shamanna SB. Maternal and Neonatal Outcome in Mothers with Gestational Diabetes Mellitus. Indian J Endocrinol Metab 2017; 21: 854-8.

12. Lapolla A, Dalfrà MG, Bonomo M, Parretti E, Mannino D, Mello G, et al. Gestational diabetes mellitus in Italy: a multicenter study. Eur J Obstet Gynecol Reprod Biol. 2009; 145: 149-53.

13. Keshavarz M, Cheung NW, Babaee GR, Moghadam HK, Ajami ME, Shariati M. Gestational diabetes in Iran: incidence, risk factors and pregnancy outcomes. Diabetes Res Clin Pract 2005; 69: 279-86.

14. Ovesen PG, Jensen DM, Damm P, Rasmussen S, Kesmodel US. Maternal and neonatal outcomes in pregnancies complicated by gestational diabetes. a nation-wide study. J Matern Neonatal Med 2015; 28: 1720-4.

15. Erem C, Kuzu UB, Deger O, Can G. Prevalence of gestational diabetes mellitus and associated risk factors in Turkish women: the Trabzon GDM Study. Arch Med Sci 2015; 11: 724-35.

16. Gascho CL, Leandro DM, Ribeiro E Silva T, Silva JC. Predictors of cesarean delivery in pregnant women with gestational diabetes mellitus. Rev Bras Ginecol Obstet 2017; 39: 60-5.

17. Chatfield J. ACOG issues guidelines on fetal macrosomia. American College of Obstetricians and Gynecologists. Am Fam Physician 2001; 64: 169-70.

18. Mack LR, Tomich PG. Gestational Diabetes: Diagnosis, Classification, and Clinical Care. Obstet Gynecol Clin North Am 2017; 44: 207-17.

19. Armson BA. Is planned cesarean childbirth a safe alternative? CMAJ 2007;176: 475-6.
20. Aghajafari F, Nagulesapillai T, Ronksley PE, Tough SC, O'Beirne M, Rabi DM. Association between maternal serum 25-hydroxyvitamin D level and pregnancy and neonatal outcomes: systematic review and meta-analysis of observational studies. BMJ 2013; 346: 1169.

21. Lu M, Xu Y, Lv L, Zhang M. Association between vitamin D status and the risk of gestational diabetes mellitus: a meta-analysis. Arch Gynecol Obstet 2016; 293: 959-66.

22. Poel YHM, Hummel P, Lips P, Stam F, van der Ploeg T, Simsek S. Vitamin D and gestational diabetes: a systematic review and metaanalysis. Eur J Intern Med 2012; 23: 465-9.

23. Wei SQ, Qi HP, Luo ZC, Fraser WD. Maternal vitamin D status and adverse pregnancy outcomes: a systematic review and metaanalysis. J Matern Fetal Neonatal Med 2013; 26: 889-99.

24. Zhang MX, Pan GT, Guo JF, Li BY, Qin LQ, Zhang ZL. Vitamin D Deficiency Increases the Risk of Gestational Diabetes Mellitus: A Meta-Analysis of Observational Studies. Nutrients 2015; 7: 836675.

25. Sablok A, Batra A, Thariani K, Batra A, Bharti R, Aggarwal AR, et al. Supplementation of vitamin $D$ in pregnancy and its correlation with feto-maternal outcome. Clin Endocrinol (Oxf) 2015; 83: 536-41.

26. Rostami M, Tehrani FR, Simbar M, Bidhendi Yarandi R, Minooee S, Hollis BW, et al. Effectiveness of Prenatal Vitamin D Deficiency Screening and Treatment Program: A Stratified Randomized Field Trial. J Clin Endocrinol Metab 2018; 103: 2936-48.

27. Palacios C, Kostiuk LK, Peña-Rosas JP. Vitamin D supplementation for women during pregnancy. Cochrane Database Syst Rev 2019; 7: CD008873.

28. Merewood A, Mehta SD, Chen TC, Bauchner H, Holick MF. Association between Vitamin D Deficiency and Primary Cesarean Section. J Clin Endocrinol Metab 2009; 94: 940-5.

29. Scholl TO, Chen X, Stein P. Maternal Vitamin D Status and Delivery by Cesarean. Nutrients 2012; 4: 319-30.

30. Asemi Z, Hashemi T, Karamali M, Samimi M, Esmaillzadeh A. Effects of vitamin D supplementation on glucose metabolism, lipid concentrations, inflammation, and oxidative stress in gestational diabetes: a double-blind randomized controlled clinical trial. Am J Clin Nutr 2013; 98: 1425-32.

31. Asemi Z, Samimi M, Tabassi Z, Shakeri H, Esmaillzadeh A. Vitamin D supplementation affects serum high-sensitivity C-reactive protein, insulin resistance, and biomarkers of oxidative stress in pregnant women. J Nutr 2013; 143: 1432-8.

32. Saha S, Saha S. A Comparison of the Changes in Gestational Weight, Body Mass Index, and Serum Vitamin D Level in Gestational Diabetes Mellitus Patients Complemented with Vitamin D in Contrast to Those Who Did Not Receive the Supplement: A Protocol for Systematic Review a. Int J Diabetes Metab 2019; 20:1-6.

33. IOM (Institute of Medicine). Dietary Reference Intakes for Calcium and Vitamin D. 2011;Washington, DC: The National Academies Press;2011.

34. Gossman W, Chauhan K, Huecker MR. Vitamin D. Stat Pearls. 2019.

35. Knabl J, Vattai A, Ye Y, Jueckstock J, Hutter S, Kainer F, et al. Role of Placental VDR Expression and Function in Common Late Pregnancy Disorders. Int J Mol Sci 2017; 18: 2340.

36. Hosseinzadeh-Shamsi-Anar M, Mozaffari-Khosravi H, Salami MA, Hadinedoushan H, Mozayan MR. The efficacy and safety of a high dose of vitamin $\mathrm{d}$ in mothers with gestational diabetes mellitus: a randomized controlled clinical trial. Iran J Med Sci 2012; 37: 159-65.

37. Li Q, Xing B. Vitamin D3-Supplemented Yogurt Drink Improves Insulin Resistance and Lipid Profiles in Women with Gestational Diabetes Mellitus: A Randomized Double Blinded Clinical Trial. Ann Nutr Metab 2016; 68: 285-90.

38. Yazdchi R, Gargari BP, Asghari-Jafarabadi M, Sahhaf F. Effects of vitamin $\mathrm{D}$ supplementation on metabolic indices and hs-CRP levels 
in gestational diabetes mellitus patients: a randomized, doubleblinded, placebo-controlled clinical trial. Nutr Res Pract 2016; 10: 328-35.

39. Asemi Z, Esmaillzadeh A. The effect of multi mineral-vitamin D supplementation on pregnancy outcomes in pregnant women at risk for Pre-eclampsia. Int J Prev Med. 2015;6:62.

40. Asemi Z, Karamali M, Esmaillzadeh A. Effects of calcium-vitamin D co-supplementation on glycaemic control, inflammation and oxidative stress in gestational diabetes: a randomised placebocontrolled trial. Diabetologia 2014; 57: 1798-806.

41. Karamali M, Bahramimoghadam S, Sharifzadeh F, Asemi Z. Magnesium-zinc-calcium-vitamin D co-supplementation improves glycemic control and markers of cardiometabolic risk in gestational diabetes: a randomized, double-blind, placebo-controlled trial. Appl Physiol Nutr Metab 2018; 43: 565-70.

42. American Diabetes Association. Diagnosis and classification of diabetes mellitus. Diabetes Care 2014; 37(Suppl 1): 81-90.

43. Rossi G; American Diabetes Association. [Diagnosis and classification of diabetes mellitus]. Recenti Prog Med 2010; 101: 274-6.

44. Liberati A, Altman DG, Tetzlaff J, Mulrow C, Gøtzsche PC, Ioannidis JP, et al. The PRISMA statement for reporting systematic reviews and meta-analyses of studies that evaluate health care interventions: explanation and elaboration. J Clin Epidemiol 2009; 62: 1-34.

45. Higgins JPT, Thomas J, Chandler J, Cumpston M, Li T, Page MJ, et al. Cochrane Handbook for Systematic Reviews of Interventions. Version 5.1.0 [updated March 2011]. [Internet]. The Cochrane Collaboration. 2011. Available from: www.cochrane-handbook.org

46. Karamali M, Asemi Z, Ahmadi-Dastjerdi M, Esmaillzadeh A. Calcium plus vitamin D supplementation affects pregnancy outcomes in gestational diabetes: randomized, double-blind, placebocontrolled trial. Public Health Nutr 2016; 19: 156-63.

47. Jamilian M, Amirani E, Asemi Z. The effects of vitamin D and probiotic co-supplementation on glucose homeostasis, inflammation, oxidative stress and pregnancy outcomes in gestational diabetes: A randomized, double-blind, placebo-controlled trial. Clin Nutr 2019; 38: 2098-105.

48. Razavi M, Jamilian M, Samimi M, Afshar Ebrahimi F, Taghizadeh M, Bekhradi R, et al. The effects of vitamin D and omega-3 fatty acids co-supplementation on biomarkers of inflammation, oxidative stress and pregnancy outcomes in patients with gestational diabetes. Nutr Metab (Lond) 2017; 14: 80.

49. Jamilian M, Mirhosseini N, Eslahi M, Bahmani F, Shokrpour M, Chamani M, et al. The effects of magnesium-zinc-calcium-vitamin D co-supplementation on biomarkers of inflammation, oxidative stress and pregnancy outcomes in gestational diabetes. BMC Pregnancy Childbirth 2019; 19: 107.

50. Asemi Z, Karamali M, Esmaillzadeh A. Favorable effects of vitamin D supplementation on pregnancy outcomes in gestational diabetes: a double blind randomized controlled clinical trial. Horm Metab Res 2015; 47: 565-70.

51. Asemi Z. Clinical trial of the effect of combined omega- 3 fatty acids and vitamin $\mathrm{D}$ supplementation compared with the placebo on metabolic profiles and pregnancy outcomes in patients with gestational diabetes. Iranian Registry of Clinical Trials. 2017 [cited 2019 Oct 18]. p. Clinical Trial Protocol. Available from: http://en.irct. ir/trial/6136

52. Atkins D, Best D, Briss PA, Eccles M, Falck-Ytter Y, Flottorp S, et al. Grading quality of evidence and strength of recommendations. BMJ 2004; 328: 1490. 